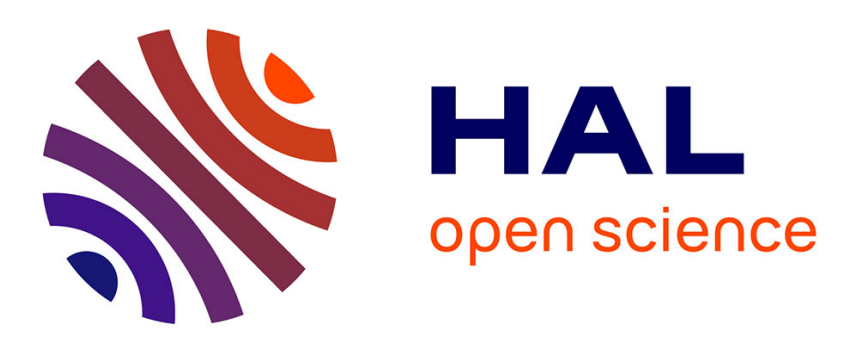

\title{
Exploring the Magnetic and Electric Side of Light through Plasmonic Nanocavities
}

\author{
Cyrine Ernandes, Hung-Ju Lin, Michel Mortier, Patrick Gredin, Mathieu \\ Mivelle, Lionel Aigouy
}

\section{- To cite this version:}

Cyrine Ernandes, Hung-Ju Lin, Michel Mortier, Patrick Gredin, Mathieu Mivelle, et al.. Exploring the Magnetic and Electric Side of Light through Plasmonic Nanocavities. Nano Letters, 2018, 18 (8), pp.5098 - 5103. 10.1021/acs.nanolett.8b01956 . hal-01872523

\section{HAL Id: hal-01872523 \\ https://hal.sorbonne-universite.fr/hal-01872523}

Submitted on 12 Sep 2018

HAL is a multi-disciplinary open access archive for the deposit and dissemination of scientific research documents, whether they are published or not. The documents may come from teaching and research institutions in France or abroad, or from public or private research centers.
L'archive ouverte pluridisciplinaire HAL, est destinée au dépôt et à la diffusion de documents scientifiques de niveau recherche, publiés ou non, émanant des établissements d'enseignement et de recherche français ou étrangers, des laboratoires publics ou privés. 


\title{
Exploring the magnetic and electric side of light through plasmonic nanocavities
}

\author{
Cyrine Ernandes ${ }^{\dagger, t}$, Hung-Ju Lin ${ }^{+}$, Michel Mortier ${ }^{\S}$, Patrick Gredin ${ }^{\S, £}$, Mathieu Mivelle ${ }^{\dagger *}$ and \\ Lionel Aigouy ** $^{*}$
}

†Sorbonne Université, CNRS, Institut des NanoSciences de Paris, UMR 7588, 75005 Paris, France

${ }^{\ddagger}$ LPEM, ESPCI Paris, PSL Research University, CNRS, Sorbonne Université, 75005 Paris, France

${ }^{\S}$ Institut de Recherche de Chimie Paris, IRCP, NSCP Chimie ParisTech, 75005 Paris, France

${ }^{£}$ Sorbonne Université, Faculté des Sciences, UFR 926, 75005, Paris, France

\begin{abstract}
Light-matter interactions are often considered to be mediated by the electric component of light only, neglecting the magnetic contribution. However, the electromagnetic energy density is equally distributed between both parts of the optical fields. Within this scope, we experimentally demonstrate here, in excellent agreement with numerical simulations, that plasmonic nanostructures can selectively manipulate and tune the magnetic versus electric emission of luminescent nanocrystals. In particular, we show selective enhancement or decay of magnetic and electric emission from trivalent europium-doped nanoparticles in the vicinity of plasmonic nanocavities, designed to efficiently couple to either the electric or magnetic emission of the quantum emitter. Specifically, by precisely controlling the spatial position of the emitter with respect to our plasmonic nanostructures, by means of a near-field optical microscope, we record local distributions of both magnetic and electric radiative local densities of states (LDOS) with nanoscale precision. The distribution of the radiative LDOS reveals the modification of both the magnetic and electric optical quantum environments induced by the presence of the metallic nanocavities. This manipulation and enhancement of magnetic lightmatter interaction by means of plasmonic nanostructures opens up new possibilities for the research fields of opto-electronics, chiral optics, nonlinear\&nano-optics, spintronics and metamaterials, amongst others.
\end{abstract}


KEYWORDS: Nano-photonics, electric and magnetic dipole transitions, luminescence enhancement, plasmonic cavities, electric and magnetic LDOS, near-field optics.

TEXT. The constant advances in nanophotonics have recently led to the development of optical nanostructures allowing to manipulate the emission of fluorescent emitters at the nanoscale. ${ }^{1-4}$ However, the underlying interactions between quantum emitters and photonic nanostructures have often been assumed to be only mediated by the optical electric field, discarding the magnetic component of light. ${ }^{5}$ Indeed, at optical frequencies, magnetic dipole (MD) transitions are at least two orders of magnitude weaker than electric dipole (ED) transitions. ${ }^{6-8}$ For this reason, most of the past studies involving single emitters (molecules ${ }^{9-11}$ or quantum dots ${ }^{12,13}$ ) have solely been focused on the modification of the excitation or emission properties of ED transitions. ${ }^{14-18}$

Recently, it was shown that strong MD transitions could as well be found in a certain class of metallic materials such as rare earth ions. ${ }^{19-23}$ Researchers have thus naturally explored the manipulation of the excitation and emission of the MD transitions from these ions. ${ }^{23-28}$ In particular, in trivalent europium $\left(\mathrm{Eu}^{3+}\right)$, it was demonstrated that by using a simple metallic surface, spontaneous emission of a MD transition could be manipulated in the same way as an ED transition in the visible range. ${ }^{21,} 29$ In particular, we previously demonstrated that by changing the distance between a nanoparticle containing electric and magnetic dipolar transitions and a gold strip of micrometer size, we could engineer their respective electric and magnetic emission, ${ }^{29}$ in the same way it was predicted and demonstrated by K. H Drexhage ${ }^{30}$ and Chance et al. ${ }^{14}$ This approach Nevertheless, in order to go further in the engineering of the interactions between magnetic light and matter, it is necessary to design nanostructures allowing to boost these interactions. ${ }^{31-49}$ Along those lines, we recently demonstrated for the first time how dielectric nanoantennas were able to enhance the magnetic emission of quantum emitters ${ }^{34}$ and researchers have demonstrated, through assemble measurements, that metallic nanostructured films could promote the transmission of magnetic emission. ${ }^{26}$ But so far, only theoretical works have studied and demonstrated the ability of single plasmonic nanostructures ${ }^{35-38}$ to manipulate the spontaneous emission of MD transitions by manipulating the magnetic LDOS at the nanoscale. 
In this article, by coupling a nanocrystal that holds strong intrinsic magnetic and electric dipole transitions to plasmonic nanostructures, we experimentally demonstrate the manipulation of magnetic and electric light emission, in excellent agreement with numerical simulations. To this end, we consider the near field interaction between plasmonic cavities and a single $\mathrm{Eu}^{3+}$ doped nanoparticle. While our previous $\operatorname{study}^{29}$ demonstrated manipulation of electric and magnetic emission in a longitudinal plane of a gold mirror, we showed in here that the luminescence associated to magnetic and electric transitions of the emitters can be modified and manipulated in the same way by changing the lateral position of the particle inside nanostructured plasmonic cavities. Moreover, by scanning the particle over the nanostructures, we recover from the experimental data the spatial distribution of the electric and magnetic relative radiative local densities of state (EMLDOS) at the surface of the cavities.

In order to find the appropriate parameters for which a plasmonic cavity can efficiently modify the electric and magnetic transitions of $\mathrm{Eu}^{3+}$ nanocrystals, we first carried out Finite Difference Time Domain (FDTD) numerical simulations to explore the evolution of the electric and magnetic branching ratios for different types of cavities (see supporting information). To simplify the problem, we focused on linear, one-dimensional structures, formed by parallel grooves in a thin metal film. We show in Figure 1a the evolution of the electric and magnetic branching ratios ((relative weight of each transition (see supporting information)) for isotropic electric and magnetic dipoles centered on a cavity of variable length. In here, the relative experimental strength of each transition is not yet considered. The highest variations of the ED and MD transitions occur for cavity lengths of $230 \mathrm{~nm}, 570 \mathrm{~nm}$ and $870 \mathrm{~nm}$, which correspond to the situation for which the nanoemitters are efficiently coupled to it. Based on these numerical simulations, we designed and nanofabricated (see supporting information) two cavities with lateral lengths of $570 \mathrm{~nm}$ and $870 \mathrm{~nm}$, corresponding to the maximum coupling of the ED and MD transitions in their centers, respectively. Figure $1 \mathrm{~b}$ depicts a scanning electron microscope (SEM) image of both plasmonic cavities (see supporting information for nanofabrication details). 


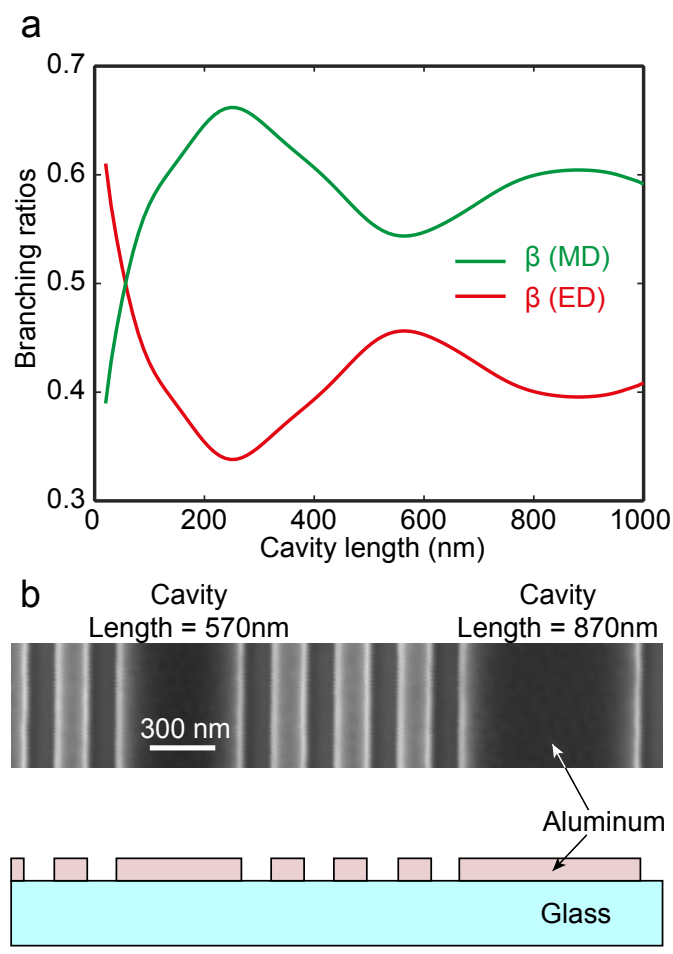

Figure 1. (a) Theoretical branching ratios associated with magnetic (green) and electric (red) isotropic dipoles, placed in the center of a cavity made of a 40 thick layer of aluminum and of variable length (see supporting information). In here, the value of $230 \mathrm{~nm}$ will not be considered in this study. Indeed, the size of the europium-doped nanocrystals (see supporting information) is only slightly smaller, so only weak spatial variations could be expected for these structures due to average effects. (b) SEM image and schematic of the nanofabricated sample. Each cavity length is indicated above the image.

To study the interaction between the plasmonic cavities and the europium emitter, we used the experimental setup shown in Figure 2a. It consists of a sharp atomic force microscope (AFM) tip with a single $\mathrm{Eu}^{3+}$ doped nanocrystal attached at its extremity (inset in Figure 2a). The tip is positioned in close proximity to the plasmonic nanostructures and both the tip and the sample are illuminated by a continuous $532 \mathrm{~nm}$ laser focused at oblique incidence (see supporting information).

The photoluminescence emission spectrum of the $\mathrm{Eu}^{3+}$ nanocrystal far away from the surface and its associated band diagram are shown in Figure $2 b$ and $c$. We can distinguish the three fundamental emission lines associated to the different electric transitions (ET) or magnetic transition (MT) in the nanocrystal: ${ }^{20}$ the MT(1) ${ }^{5} \mathrm{D}_{0} \rightarrow{ }^{7} \mathrm{~F}_{1}$ from 580-600nm, the ET(2) ${ }^{5} \mathrm{D}_{0}$ 
$\rightarrow{ }^{7} \mathrm{~F}_{2}$ from $600-625 \mathrm{~nm}$ and $\mathrm{ET}(3){ }^{5} \mathrm{D}_{0} \rightarrow{ }^{7} \mathrm{~F}_{4}$ from $685-705 \mathrm{~nm}$. It is important to mention that, for the present work we will ignore the contribution of the last ET (3). Since it is well separated from the higher energy ones, it was found to be weakly influenced by the environment, as observed in previous studies. ${ }^{29}$ And because we chose to focus mainly on the spectrally close magnetic (MT(1)) and electric (ET(2)) dipolar transitions, in this study, all other decay channels from ${ }^{5} \mathrm{D}_{0}$ to the ground state, including ET(3), are considered as losses. Using this spectrum recorded in far field, we then calculated the effective values of the oscillator strengths of MT (1) and ET (2), found to be respectively 0.33 and 0.67 . These values will be then considered later on in the simulations, in order to quantitatively compare our experimental and theoretical results. 

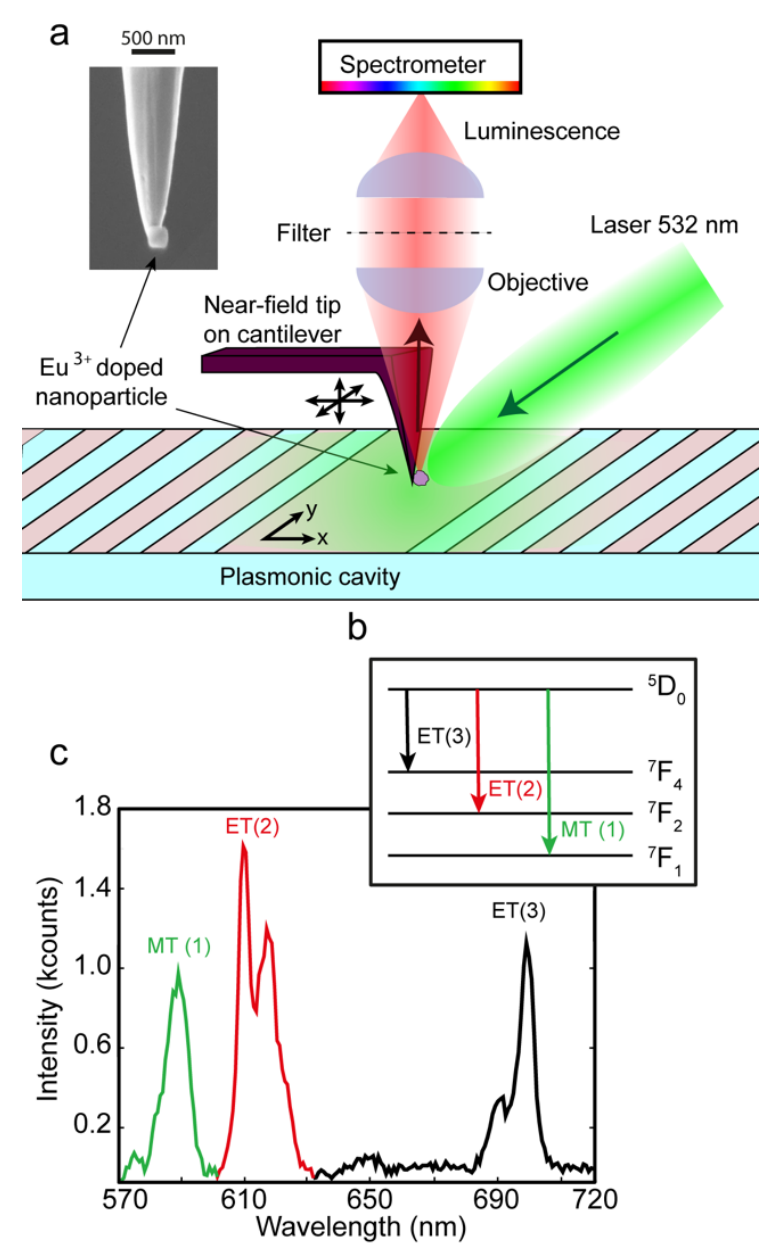

Figure 2. (a) Schematic of the near field experimental setup. An AFM tip holding a single $\mathrm{Eu}^{3+}$ doped nanoparticle (150-200 $\mathrm{nm}$ diameter, see Figure S1 in the supporting information) is scanning a plasmonic nanocavity at a constant distance of about $20 \mathrm{~nm}$. Both the particle and the cavity are excited by a $532 \mathrm{~nm}$ laser, and for each position of the emitter with respect to the nanostructure, a spectrometer is recording the emission spectrum of the nanosource. Inset: SEM image of a single nanocrystal doped with $\mathrm{Eu}^{3+}$ and attached at the apex of a tungsten AFM tip. (b) Partial band diagram of the $\mathrm{Eu}^{3+}$ ions. (c) Emission spectrum of the $\mathrm{Eu}^{3+}$ doped nanoparticle recorded in far field.

Using the setup described in Figure 2a, we then scanned the cavities shown in Figure 1b with a $\mathrm{Eu}^{3+}$-doped nanoparticle and measured its emission spectrum at every position above the structures. Before quantitative analyzing the variations of each photoluminescence lines separately, we show in Figure $3 \mathrm{a}$ and $\mathrm{c}$ the evolution of the distribution of the total nanocrystal photoluminescence on the surface of the cavities. The excitation being made through an electric dipolar transition $(\lambda=532 \mathrm{~nm}),{ }^{24}$ these distributions are strongly related to the intensity of the 
electric near field located at the surface of the cavity and exciting the particle at this wavelength. This is corroborated by numerical simulations displayed in Figure $3 \mathrm{~b}$ and $\mathrm{d}$ and obtained by exciting the cavities at $\lambda=532 \mathrm{~nm}$ by an incident plane wave launched from the upper side of the cavities, with an angle of 70 degrees with respect to the normal of the sample and with a TM polarization. The variations of the collected signal along the scanned area are in fairly good agreement with the simulations, and the maxima and minima of photoluminescence correspond well to the ones of the total theoretical electric field intensity. However, there are some differences, which can be attributed to the presence of the tip and the particle which were both not taken into account in the simulations. The observed optical oscillations indicate that the particle is placed in close enough proximity to the surface since it senses the stationary surface plasmon waves located inside the cavities. In Figure $3 \mathrm{e}$ and $3 \mathrm{f}$, we show the normalized emission spectra of the nanocrystal at two different positions of the cavities indicated by colored dots in Figure 3a and 3c. These spectra exhibit two peaks associated to the magnetic (labeled by an $\mathrm{M}$ ) and electric (labeled by an E) dipole transitions of the $\mathrm{Eu}^{3+}$, respectively. As it can be seen, the spectra do not overlap perfectly according to the particle position inside the cavities. This result indicates that the relative importance of the magnetic and electric dipolar transitions (MT and ET) of the europium ions can manipulate only by changing the particle position inside the nanostructures. 

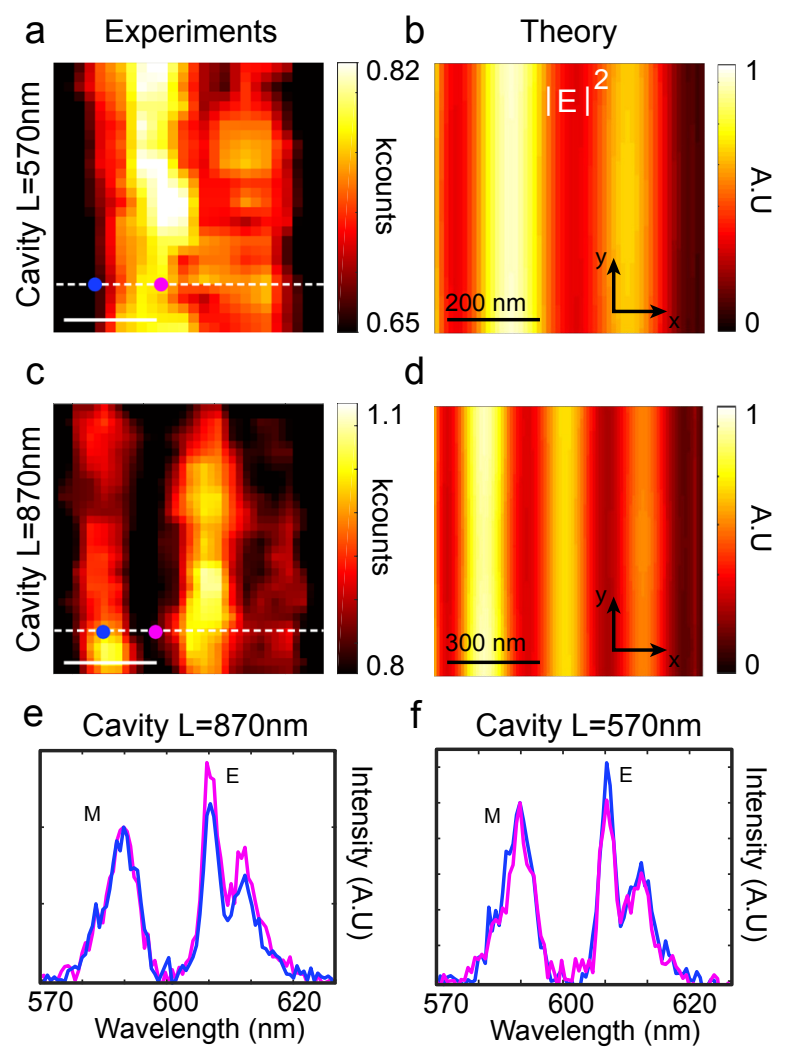

Figure 3. Two-dimensional distribution of the total collected photoluminescence $(\mathrm{a}, \mathrm{c})$ and total simulated electric intensity $(\lambda=532 \mathrm{~nm})(\mathrm{b}, \mathrm{d})$ in the cavities plane, for the cavity lengths of $570 \mathrm{~nm}(\mathrm{a}, \mathrm{b})$ and $870 \mathrm{~nm}(\mathrm{c}, \mathrm{d})$. (e,f) Normalized emission spectra of MT (1) and ET (2) measured at two different positions inside each cavity lengths, (e) $570 \mathrm{~nm}$ and (f) $870 \mathrm{~nm}$, in each case along the same transversal dashed line and indicated by blue and pink dots in $(\mathrm{a}, \mathrm{c})$.

To evaluate the relative contribution of the electric and magnetic transitions to the total luminescence, we then calculated the branching ratios $\left(\beta^{\mathrm{e}, \mathrm{m}}\right)$ associated to these transitions. Experimentally, the relative weight of the $\mathrm{MT}\left(\beta^{\mathrm{m}}\right)$ and $\mathrm{ET}\left(\beta^{\mathrm{e}}\right)$ along the plasmonic structures are calculated as: ${ }^{25,29}$

$\beta^{\mathrm{e}, \mathrm{m}}(\mathrm{p})=\frac{\mathrm{I}^{\mathrm{e}, \mathrm{m}}(\mathrm{p})}{\mathrm{I}_{\text {tot }}(\mathrm{p})}$

where $\mathrm{p}$ is the position of the particle along the cavity, $\mathrm{I}^{\mathrm{e}, \mathrm{m}}$ is the luminescence intensity of the electric $\left(\mathrm{I}^{\mathrm{e}}\right)$ or magnetic $\left(\mathrm{I}^{\mathrm{m}}\right)$ transition and $\mathrm{I}_{\text {tot }}$ is the total luminescence intensity. 
We show in Figure 4a and c the evolution of the experimental electric and magnetic branching ratios for both cavities. These curves were obtained by averaging 20 scans along the plasmonic nanostructures (x-direction). The corresponding theoretical calculations of the branching ratios are displayed in Figure $4 \mathrm{~b}$ and $\mathrm{d}$ (see supporting information). We can observe an excellent agreement between the numerical simulations and the experimental data: the spatial oscillations, the maxima and the minima of $\beta^{\mathrm{e}}$ and $\beta^{\mathrm{m}}$ are all located, for each case, at the same positions inside the cavities. Meanwhile, in accordance with the result displayed in Figure 1, the small cavity exhibits a maximum value of $\beta^{\mathrm{e}}$ in its center whereas the large cavity shows an opposite behavior, namely, a maximum value of $\beta^{\mathrm{m}}$ in the center (see supporting information for more examples). The small differences in amplitude between the experiments and the simulations are attributed to the presence of the tip inside the cavities, which might slightly change their behavior. Another example shown in Figure S3, made with another tip and particle but in the same experimental conditions, exhibits as well a variability in the amplitude with respect to Figure 4. This indicates that the probe is indeed most likely the variable factor causing this fluctuation. 


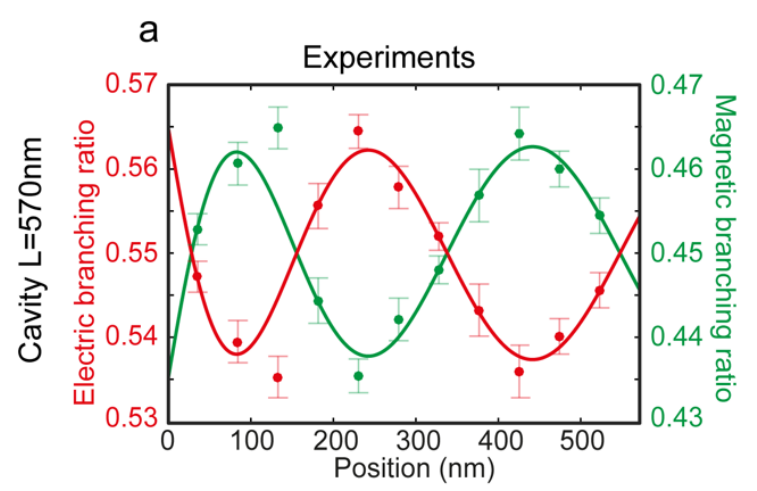

C

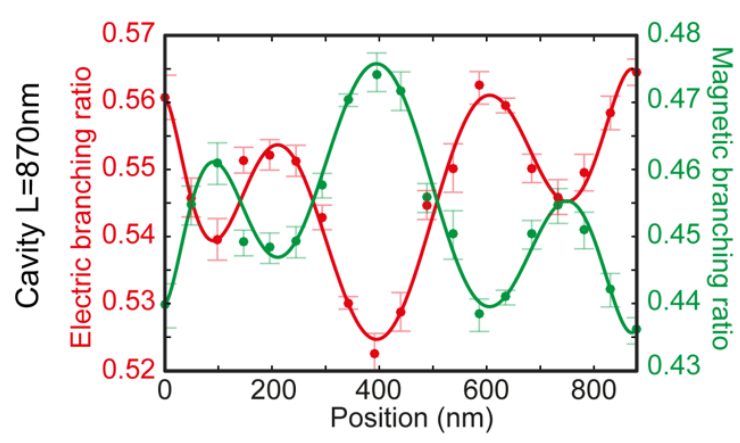

b

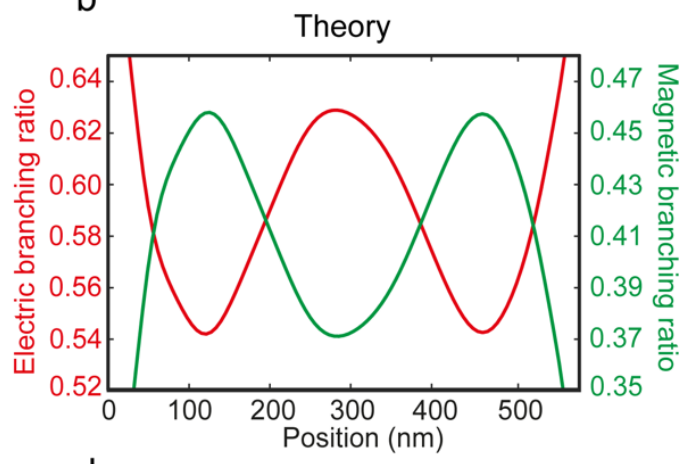

d

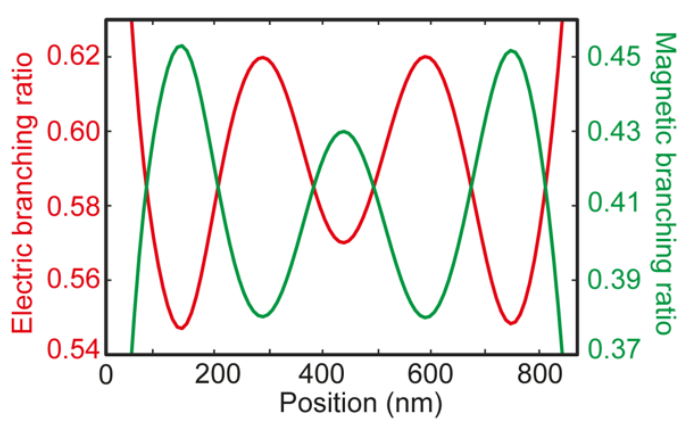

Figure 4. Magnetic and electric branching ratios. (a,c) Experimental branching ratios associated with magnetic (green) and electric (red) transitions of the $\mathrm{Eu}^{3+}$ according to the nanoparticle positions inside the $570 \mathrm{~nm}$ (a) and $870 \mathrm{~nm}$ (c) long cavities. The red and green dots represent the experimental data, the full line is a polynomial fit and the error bars represent the standard error of the mean. $(b, d)$ Numerical counterparts of $(a, c)$ respectively.

These results clearly demonstrate that the electric and magnetic emission of trivalent europium ions can be manipulated, in a controlled manner, by means of plasmonic nanostructures. The different emission behaviors of the electric and magnetic transitions, given by the branching ratios, are due to the non-spatial overlap of the electric and magnetic radiative LDOS at the cavities surface. To illustrate this relationship, we calculated the relative contribution of the electric and magnetic components of the radiative LDOS using the values of the experimental branching ratio, as we previously described in: ${ }^{29}$

$\rho^{\mathrm{e}, \mathrm{m}}=\frac{\beta^{\mathrm{e}, \mathrm{m}} \cdot \mathrm{f}^{\mathrm{m}, \mathrm{e}}}{\beta^{\mathrm{e}, \mathrm{m}} \cdot \mathrm{f}^{\mathrm{m}, \mathrm{e}}+\beta^{\mathrm{m}, \mathrm{e}} \cdot \mathrm{f}^{\mathrm{e}, \mathrm{m}}}$ 
where $\rho^{\mathrm{e}, \mathrm{m}}$ are the electric $\left(\rho^{\mathrm{e}}\right)$ or magnetic $\left(\rho^{\mathrm{m}}\right)$ relative radiative LDOS and $\mathrm{f}^{\mathrm{e}, \mathrm{m}}$ are the oscillator strengths for the electric $\left(\mathrm{f}^{\mathrm{e}}=0.67\right)$ or magnetic $\left(\mathrm{f}^{\mathrm{m}}=0.33\right)$ transitions respectively (calculated in far field (Figure 2c)).

The spatial distributions of the radiative magnetic local density of state (further referred as MLDOS) and the radiative electric one (further referred as ELDOS) in the cavities plane are displayed in Figure 5 a-d. As expected, the ELDOS and MLDOS do not overlap in the cavities plane, and exhibit several maxima and minima situated at different positions. In Figure 5e and $5 f$, we show the evolution of the experimental ELDOS and MLDOS by averaging 20 scans along the cavities. Although these curves seem similar to those already shown in Figure 4a and $4 \mathrm{c}$, they represent the electric and magnetic radiative LDOS inside the cavities and are independent of the oscillator strengths of the transitions and therefore from the emitter considered. Thus, they are directly related to the electric and magnetic optical quantum environment of the nanocavities.

These results highlight the ability of such nanostructures to influence and tune the emission of rare-earth ions, by maximizing or minimizing either the electric or magnetic LDOS at different locations in their near-field. 

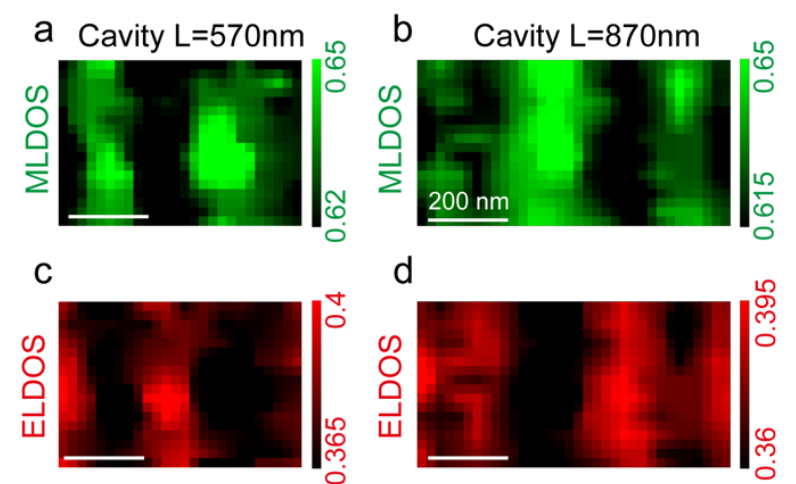

d
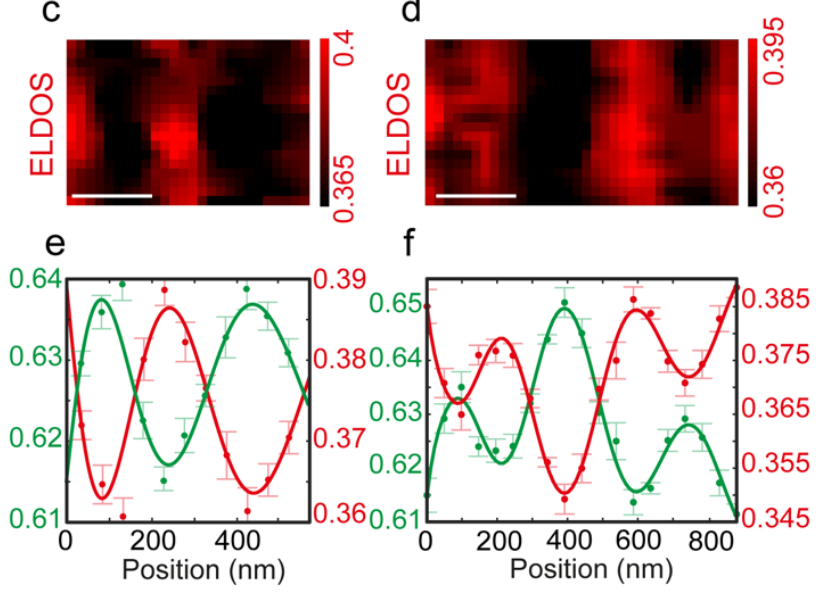

Figure 5. Radiative local density of state. 2D experimental maps of the radiatives (a,b) MLDOS and (c,d) ELDOS in the plane above the $570 \mathrm{~nm}(\mathrm{a}, \mathrm{c})$ and $870 \mathrm{~nm}(\mathrm{~b}, \mathrm{~d})$ long cavities. (e,f) Experimental magnetic (green) and electric (red) radiative LDOS for each cavity lengths, (e) $570 \mathrm{~nm}$ and (f) $870 \mathrm{~nm}$. Dots are the experimental data, full lines represent a polynomial fit and the error bars are the standard error of the mean.

In summary, we demonstrated experimentally, in excellent agreement with numerical simulations, that the emission of a single $\mathrm{Eu}^{3+}$ doped nanocrystal that contains magnetic and electric transitions, can be perfectly manipulated and controlled by plasmonic cavities. According to the position of the trivalent europium nanoparticle inside the nanostructures, we showed that both ED and MD transitions emission can be manipulated with an increase, or alternatively a decrease, of their respective near-field luminescence. Furthermore, we retrieved the spatial distribution of radiative electric and magnetic parts of the local density of state at the surface of the cavities, which is a fundamental quantum characteristic of these structures. This study shows that the magnetic part of light can be engineered in the same way as its electric counterpart using metallic nanostructures and we are confident that this work will pave the way for the design of new plasmonic geometries enabling the boost of magnetic light and matter interactions, allowing applications in fields as diverse as spintronics, photochemistry, ${ }^{50}$ sensing, ${ }^{51}$ molecular chirality ${ }^{52}$ amongst others. 


\section{ASSOCIATED CONTENT}

\section{Supporting Information.}

Nanocrystal synthesis. Experimental set-up and sample fabrication. Numerical calculation. SEM image of the doped nanoparticles. Branching ratios versus the distance to the sample. Additional branching ratios inside the cavities.

\section{AUTHOR INFORMATION}

\section{Corresponding Authors}

*E-mail: mathieu.mivelle@,insp.upmc.fr lionel.aigouy@espci.fr

\section{Author Contributions}

M.Mi. and L.A. designed the research. M.Mi. designed, simulated and fabricated the antennas. M.Mo. and P.G. synthesized the trivalent europium doped nanocrystals. C.E, H.J.L. and L.A. performed the measurements and analyzed the data. C.E., M.Mi. and L.A. wrote the manuscript with input from all authors.

The authors declare no competing financial interest.

\section{ACKNOWLEDGMENTS}

The authors thank Maria F. Garcia-Parajo for fruitful discussions and her help on the nanostructuration of the sample. M.M acknowledges support from the French “Investissements d'Avenir" program (Labex MATISSE), the DIM Nano-K program from "Région Ile de France", the CNRS emergence program and the ANR tremplin under reference ANR-17-ERC3-0006-01. 


\section{REFERENCES}

1. Anger, P.; Bharadwaj, P.; Novotny, L. Physical review letters 2006, 96, 11, 113002.

2. Lodahl, P.; Van Driel, A. F.; Nikolaev, I. S.; Irman, A.; Overgaag, K.; Vanmaekelbergh, D.; Vos, W.

L. Nature 2004, 430, 7000, 654.

3. Vahala, K. J. nature 2003, 424, 6950, 839.

4. Novotny, L.; Van Hulst, N. Nature photonics 2011, 5, 2, 83.

5. Kühn, S.; Håkanson, U.; Rogobete, L.; Sandoghdar, V. Physical review letters 2006, 97, 1, 017402.

6. Cowan, R. D., The theory of atomic structure and spectra. Univ of California Press: 1981.

7. Giessen, H.; Vogelgesang, R. Science 2009, 326, 5952, 529-530.

8. Burresi, M.; Van Oosten, D.; Kampfrath, T.; Schoenmaker, H.; Heideman, R.; Leinse, A.; Kuipers, L. Science 2009, 326, 5952, 550-553.

9. Tam, F.; Goodrich, G. P.; Johnson, B. R.; Halas, N. J. Nano letters 2007, 7, 2, 496-501.

10. Taminiau, T.; Stefani, F.; Segerink, F. B.; Van Hulst, N. Nature Photonics 2008, 2, 4, 234.

11. Kinkhabwala, A.; Yu, Z.; Fan, S.; Avlasevich, Y.; Müllen, K.; Moerner, W. Nature Photonics 2009, 3, 11, 654 .

12. Farahani, J. N.; Pohl, D. W.; Eisler, H.-J.; Hecht, B. Physical review letters 2005, 95, 1, 017402.

13. Song, J.-H.; Atay, T.; Shi, S.; Urabe, H.; Nurmikko, A. V. Nano letters 2005, 5, 8, 1557-1561.

14. Chance, R.; Prock, A.; Silbey, R. Adv. Chem. Phys 1978, 37, 1, 65.

15. Bharadwaj, P.; Novotny, L. Optics Express 2007, 15, 21, 14266-14274.

16. Bouchet, D.; Mivelle, M.; Proust, J.; Gallas, B.; Ozerov, I.; Garcia-Parajo, M. F.; Gulinatti, A.; Rech, I.; De Wilde, Y.; Bonod, N. Physical Review Applied 2016, 6, 6, 064016.

17. Regmi, R.; Berthelot, J.; Winkler, P. M.; Mivelle, M.; Proust, J.; Bedu, F. d. r.; Ozerov, I.; Begou, T.; Lumeau, J.; Rigneault, H. Nano letters 2016, 16, 8, 5143-5151.

18. Klimov, V. V. e.; Ducloy, M.; Letokhov, V. S. Quantum electronics 2001, 31, 7, 569-586.

19. Noginova, N.; Barnakov, Y.; Li, H.; Noginov, M. Optics express 2009, 17, 13, 10767-10772.

20. Karaveli, S.; Zia, R. Optics letters 2010, 35, 20, 3318-3320.

21. Karaveli, S.; Zia, R. Physical review letters 2011, 106, 19, 193004.

22. Karaveli, S.; Wang, S.; Xiao, G.; Zia, R. ACS nano 2013, 7, 8, 7165-7172.

23. Choi, B.; Iwanaga, M.; Sugimoto, Y.; Sakoda, K.; Miyazaki, H. T. Nano letters 2016, 16, 8, 51915196.

24. Kasperczyk, M.; Person, S.; Ananias, D.; Carlos, L. D.; Novotny, L. Physical review letters 2015, 114, 16, 163903.

25. Rabouw, F. T.; Prins, P. T.; Norris, D. J. Nano letters 2016, 16, 11, 7254-7260.

26. Hussain, R.; Kruk, S. S.; Bonner, C. E.; Noginov, M. A.; Staude, I.; Kivshar, Y. S.; Noginova, N.; Neshev, D. N. Optics letters 2015, 40, 8, 1659-1662.

27. Li, D.; Jiang, M.; Cueff, S.; Dodson, C. M.; Karaveli, S.; Zia, R. Physical Review B 2014, 89, 16, 161409.

28. Taminiau, T. H.; Karaveli, S.; Van Hulst, N. F.; Zia, R. Nature communications 2012, 3, 979.

29. Aigouy, L.; Cazé, A.; Gredin, P.; Mortier, M.; Carminati, R. Physical review letters 2014, 113, 7, 076101.

30. Drexhage, K. H., IV interaction of light with monomolecular dye layers. In Progress in optics, Elsevier: 1974; Vol. 12, pp 163-232.

31. Baranov, D. G.; Savelev, R. S.; Li, S. V.; Krasnok, A. E.; Alù, A. Laser \& Photonics Reviews 2017.

32. García-Etxarri, A.; Gómez-Medina, R.; Froufe-Pérez, L. S.; López, C.; Chantada, L.; Scheffold, F.; Aizpurua, J.; Nieto-Vesperinas, M.; Sáenz, J. J. Optics express 2011, 19, 6, 4815-4826.

33. Rolly, B.; Bebey, B.; Bidault, S.; Stout, B.; Bonod, N. Physical Review B 2012, 85, $24,245432$. 
34. Sanz-Paz, M.; Ernandes, C.; Esparza, J. U.; Burr, G. W.; van Hulst, N. F.; Maître, A.; Aigouy, L.; Gacoin, T.; Bonod, N.; Garcia-Parajo, M. F. Nano Letters 2018.

35. Mivelle, M.; Grosjean, T.; Burr, G. W.; Fischer, U. C.; Garcia-Parajo, M. F. ACS Photonics 2015, 2, 8, 1071-1076.

36. Chigrin, D. N.; Kumar, D.; Cuma, D.; von Plessen, G. ACS Photonics 2015, 3, 1, 27-34.

37. Hein, S. M.; Giessen, H. Physical review letters 2013, 111, 2, 026803.

38. Grosjean, T.; Mivelle, M.; Baida, F.; Burr, G.; Fischer, U. Nano letters 2011, 11, 3, 1009-1013.

39. Li, J.; Verellen, N.; Van Dorpe, P. ACS Photonics 2017, 4, 8, 1893-1898.

40. Cihan, A. F.; Curto, A. G.; Raza, S.; Kik, P. G.; Brongersma, M. L. arXiv preprint arXiv:1709.04999

2017.

41. van de Haar, M. A.; van de Groep, J.; Brenny, B. J.; Polman, A. Optics Express 2016, 24, 3, 20472064.

42. Kuznetsov, A. I.; Miroshnichenko, A. E.; Brongersma, M. L.; Kivshar, Y. S.; Luk'yanchuk, B. Science 2016, 354, 6314, aag2472.

43. Feng, T.; Xu, Y.; Liang, Z.; Zhang, W. Optics letters 2016, 41, 21, 5011-5014.

44. Evlyukhin, A. B.; Reinhardt, C.; Seidel, A.; Luk'yanchuk, B. S.; Chichkov, B. N. Physical Review B 2010, 82, 4, 045404.

45. Evlyukhin, A. B.; Novikov, S. M.; Zywietz, U.; Eriksen, R. L.; Reinhardt, C.; Bozhevolnyi, S. I.; Chichkov, B. N. Nano letters 2012, 12, 7, 3749-3755.

46. Schmidt, M. K.; Esteban, R.; Sáenz, J.; Suárez-Lacalle, I.; Mackowski, S.; Aizpurua, J. Optics express 2012, 20, 13, 13636-13650.

47. Albella, P.; Poyli, M. A.; Schmidt, M. K.; Maier, S. A.; Moreno, F.; Sáenz, J. J.; Aizpurua, J. The Journal of Physical Chemistry C 2013, 117, 26, 13573-13584.

48. Coenen, T.; Van De Groep, J.; Polman, A. ACS nano 2013, 7, 2, 1689-1698.

49. Zambrana-Puyalto, X.; Bonod, N. Physical Review B 2015, 91, 19, 195422.

50. Manjavacas, A.; Fenollosa, R.; Rodriguez, I.; Jiménez, M. C.; Miranda, M. A.; Meseguer, F. Journal of Materials Chemistry C 2017, 5, 45, 11824-11831.

51. Xi, Z.; Urbach, H. Physical review letters 2017, 119, 5, 053902.

52. Valev, V. K.; Govorov, A. O.; Pendry, J. Advanced Optical Materials 2017, 5, 16.

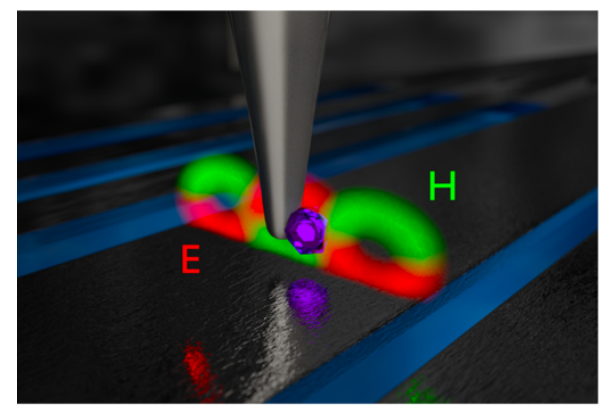

TABLE OF CONTENTS GRAPHIC 